\title{
Microbes, Mathematics, and Models
}

*Maureen A. O'Malley

University of Bordeaux, France; University of Sydney, Australia

Email: maureen.omalley@sydney.edu.au

Address: HPS, Carslaw Building, University of Sydney, NSW 2006, Australia

*Emily C. Parke

University of Auckland, New Zealand

Email: e.parke@auckland.ac.nz

Address: Department of Philosophy, Room 538, Level 5, 14A Symonds St

University of Auckland, Auckland 1010, New Zealand

${ }^{*}$ Equal co-authors; both corresponding authors

\begin{abstract}
Microbial model systems have a long history of fruitful use in fields that include evolution and ecology. In order to develop further insight into modelling practice, we examine how the competitive exclusion and coexistence of competing species have been modelled mathematically and materially over the course of a long research history. In particular, we investigate how microbial models of these dynamics interact with mathematical or computational models of the same phenomena. Our cases illuminate the ways in which microbial systems and equations work as models, and what happens when they generate inconsistent findings about shared targets. We reveal an iterative strategy of comparative modelling in different media, and suggest reasons why microbial models have a special degree of epistemic tractability in multimodel inquiry.
\end{abstract}

\section{Keywords}

Models; experimental systems; microbial model systems; competitive exclusion; coexistence; robustness analysis 


\section{Introduction}

Understanding what models are and how they work is central to philosophical work on a variety of sciences. A special feature of the life sciences is that they use organisms as material models of target phenomena, which include evolutionary, ecological, genetic, and physiological processes. Groups of organisms are constructed in order to function as experimental systems that allow the control and manipulation of represented phenomena. Because of the importance of tractability in such systems, microorganisms - prokaryotic and eukaryotic microbes, plus their viruses - have been favoured biological models for many research programmes. Microbial model systems have deep historical roots and their use has generated many major findings (Buckling et al., 2009; Garland and Rose, 2009; Jessup et al., 2004).

Philosophers have examined several aspects of the experimental evolutionary research done with bacterial microorganisms. These analyses have focused primarily on what these experiments say about evolutionary processes and theories (for example, Beatty, 2006; Plutynksi, 2001; Desjardins, 2011; Parke, 2014). However, there are other avenues of philosophical insight afforded by experimental evolutionary research, and one of them is about the nature of modelling itself (Weber, 2014). Analysing the ways in which microbial systems generate knowledge sheds light on how material or concrete models function more generally, and on how they work together with other sorts of models. These are aspects of philosophy of modelling that are not yet fully developed.

There now exists a considerable philosophical literature on model organisms that is concerned with what defines them and how they are used (e.g., Rheinberger 2010; Ankeny and Leonelli, 2011; Levy and Currie, 2015). There is a tendency in both philosophical and scientific circles to think of organismal models as closer to scientists' target phenomena than mathematical or computational models of the same target. Such a view is on display, for example, in Levy and Currie's (2015) discussion of model organisms. They contrast organismal models to other kinds of theoretical models, and suggest the former are specimens of broader classes in nature. Anecdotally, we have been told that organisms are "just too complex" to turn into full-blown models, and that organismal model systems really function as tests of mathematical models. At the very least, this position asserts that there is a hierarchy of models in terms of degree of closeness to target phenomena. Biological models-if not plucked directly from natureare regarded as samples of, or on a par with, scientists' targets in the natural world in a way that other material models typically are not, and mathematical and computational models even less so.

We will argue against this broad intuition and suggest that microbial modelling practice offers particularly clear evidence against it. To do so, we offer illustrations from a lineage rich in multimodel comparison. These examples strengthen the case for the practical and epistemic interchangeability of model media, whether mathematical or organismal. We are not saying they are always so interchangeable, but that our cases illustrate they can be, and that this finding tells against a hierarchical view of model types. 
In what follows, we focus on how microbial model systems are used in evolutionary biology and ecology, and specifically, on models of competitive exclusion and its opposite state, coexistence. Competitive exclusion is the process by which one population comes to dominate the use of a shared resource, at the expense of other populations. Coexistence refers to species that manage to live together in the same environment, despite competing for the same resources. We will show how this research area offers a particularly fruitful lens through which to examine how microbial and mathematical models work together. To do this, we sketch a historical trajectory of model-based research into these dynamics, then examine it within the framework provided by philosophy of modelling and its distinctions between different types of models. In particular, we elaborate on the different ways in which microbial model systems have been put to work in conjunction with mathematical and computational models to explain unexpected coexistence rather than predicted competitive exclusion. Our analysis clarifies how microorganisms function as model systems, and how they interact with classic mathematical models as well as computational simulations. We discuss how dependent model comparisons contribute to robustness analysis. Finally, we address why microbes are particularly epistemically tractable as material representations of biological phenomena.

\section{Philosophy of Modelling}

Philosophy of modelling commonly distinguishes between mathematical or computational models and material (or concrete or physical) models. Equations serve as the structural basis of mathematical models, algorithms form computational models, and physical structures of various sorts are used to construct material models (Weisberg, 2013; Downes, 1992; Griesemer, 1990). In our discussion, differences between mathematical and computational models are not important because we are primarily concerned with how material models are compared to models constructed from non-material mathematical media. ${ }^{1}$ We focus on how organisms, and particularly microorganisms, function as a special kind of material model.

It might seem at first glance that physical systems, whether living or non-living, just cannot model in the same way that abstract equations and algorithms can. There has been a great deal of discussion about how models - especially computer simulations - are like and unlike experiments (see Guala, 2002; Mäki, 2005; Morgan, 2005; Parker, 2009; Winsberg, 2009; Parke, 2014). An associated point of contention is whether laboratory constructions, such as the microorganismal ones we discuss, should be categorized as experimental systems or material models. These terms are often used interchangeably in the biological literature discussing these systems and their use in evolution and ecology (see, for example, Garland and Rose, 2009; Prosser et al., 2007). In philosophical

\footnotetext{
${ }^{1}$ Mathematical and computational models are not always recognized as fully distinct from one another because they are both fundamentally mathematical operations. Nevertheless, says philosopher of modelling Michael Weisberg, "they function differently in practice and have different representational capacities", so are well worth distinguishing (Weisberg, 2013, p. 20). Tarja Knuuttila (2016, p. 269), on the other hand, objects to strong distinctions being made between abstract (mathematical) models and material systems because they serve similar epistemic functions.
} 
discussions, however, including those just cited, categorization as an experimental system rather than as a model often implies different treatment, in terms of ontology, methodology, or epistemic value. The crucial epistemic point in these debates is whether biological and other physical model systems are merely components of experiments. Experiments in this view are procedures carried out with experimental objects, meaning that experimental constructs are not models. This is not our view.

From a general perspective, experimental objects of study share many similarities with material models because of the ways in which scientists use them (Rheinberger, 2010; Frigg and Nguyen, 2017). Both involve studying a physical system that stands in for a scientist's ultimate target of investigation in the natural world. Both experimental systems and models (material and otherwise) simplify complex target phenomena; both idealize; both are forced into tradeoffs between realism, precision and generality (Levins, 1966; Rainey et al. 2000; Jessup et al., 2005; Matthewson, 2011; Inkpen, 2016). Rachel Ankeny and Sabina Leonelli (2011) suggest that experimental organisms (the larger class of surrogates to which model organisms belong) have less representational scope than genuine model organisms, largely in virtue of the communities that drive the use of 'official' model organisms. The history of microorganismal modelling that we examine, however, does not make this distinction even when it enters the current era of institutionally designated model organisms.

Section 3 illustrates how microorganismal experiments play over several decades what appear to be genuine modelling roles in ecological and evolutionary population biology. Because of the experimental-modelling overlap in how organismal systems are used and referred to in scientific practice, we too use 'experimental model system' interchangeably with 'material model' or 'organismal model' to mean the systems constructed and studied by scientists in the laboratory with the aim of understanding biological systems and phenomena more generally. But as we will make clear, we do not think (micro)organismal models are closer to the target system: they are epistemically tractable material surrogates for those systems. We also bite the bullet about whether many experimental constructions should properly be regarded as models. We say they can be, on many understandings of what constitutes a model, in virtue of how they abstract, simplify and idealize the target systems they are surrogates for.

Even when there is agreement on organisms being able to play the role of material models in specified circumstances, there are still a number of questions about how these (micro)organismal models carry out their surrogacy role, and what the relationship is between the different modes of representation enabled by distinct modelling strategies. Many recent philosophical discussions of modelling have tended to emphasize the relationship between models and targets in the natural world (Figure 1, right-hand arrows). When different modelling media (for example, mathematical and material) are used to gain insight into the same targets, one model is typically treated as 'remodelling' the target in a different medium from the other model, or even more simply as just another independent instance of the back-and-forth movement between model and target. The series of mathematical and material models of the dynamics of competitive exclusion and coexistence we discuss in Section 3 do not fit this description. We show instead how modelling practice often involves moving between at least two kinds of models, with the aim of evaluating and refining each of them in light of insights gleaned from studying the 
other. Material models, as much as any other model type, are assessed against other models built in different media. We label this dependent interaction 'comparative modelling' (Figure 1, left-hand side). ${ }^{2}$

Figure 1: Relationships Between Models

Much philosophy of modelling focuses on model-target relationships, and holds that models indirectly represent target systems, as shown by the righthand arrows (for example, Giere, 1988; Godfrey-Smith, 2006; Weisberg, 2013; for alternative views see Knuuttila and Loettgers, 2016; Levy, forthcoming). ${ }^{3}$ Our case study emphasizes the importance of model-model relationships (left-hand arrows) in the practice of modelling, and demonstrates a variety of ways in which one type of model is used to assess and develop another over the course of a research program.

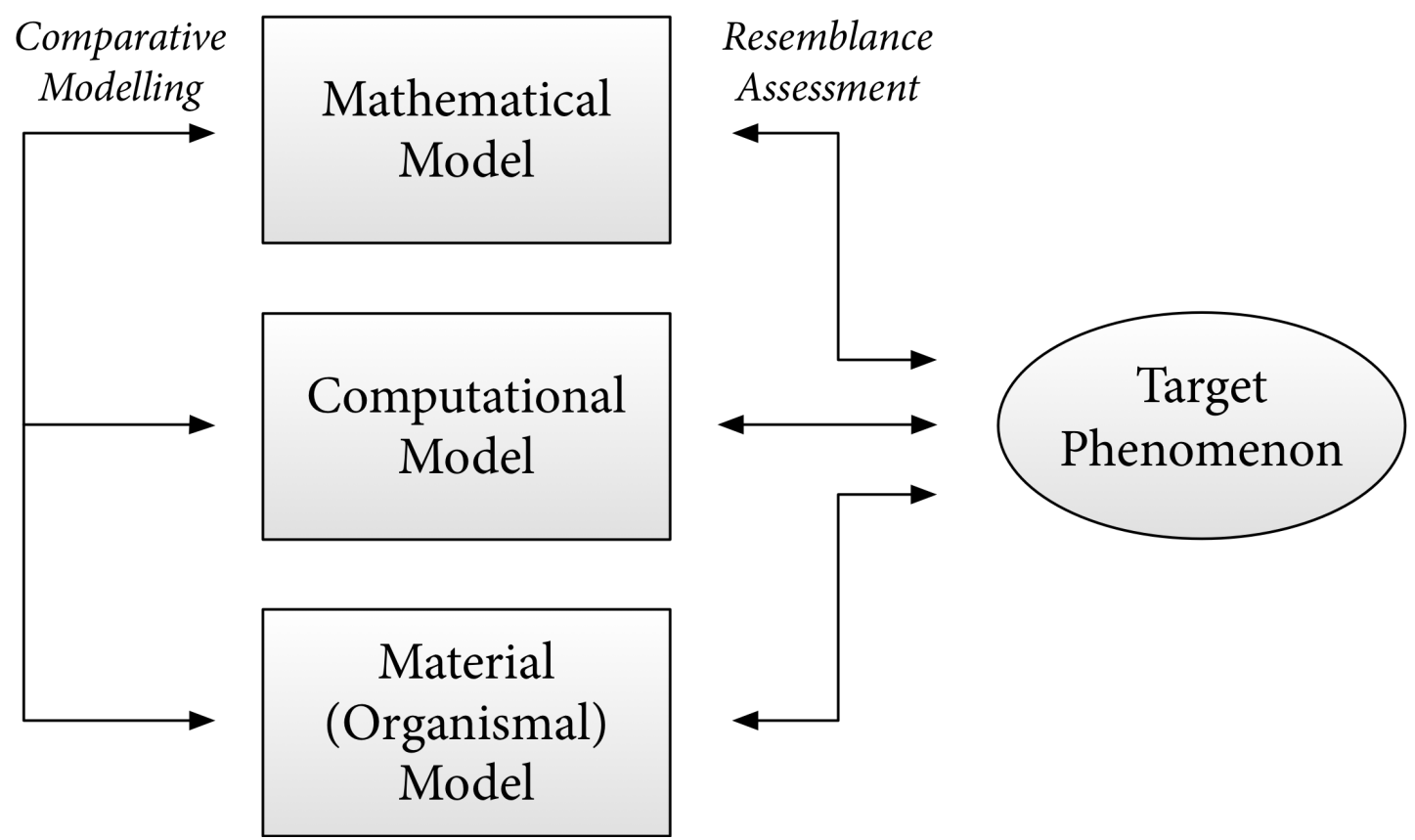

Sometimes, researchers state that mathematical or computational models are properly validated by testing their predictions in experimental model systems. Such statements might indicate that organismal systems are the target phenomena themselves. We will discuss some claims about this practice in Section 4. But material models are not merely sources of empirical data that allow the evaluation of how well mathematical or computational models fit the target system. The performance of material models is directly compared to mathematical or computational models, and vice-versa (Figure 1, left-hand arrows). Our cases show how these dynamics have played out over several decades of

${ }^{2}$ We are grateful to Brett Calcott for suggesting this term.

3 The models-as-mediators approach (Morrison and Morgan, 1999) also fits this diagram, although it would emphasize an additional layer of theory above any model, with the model navigating 'independently' between theory and target ('world'). Because this three-level scheme does not fit how microbial modellers describe their activities, we focus on the twolayer approach to models and target phenomena. 
microbial modelling of competitive exclusion and coexistence, and how modellers themselves have conceptualized this interaction. These dependent interactions between models add new practical dimensions to how robustness analysis can be understood across multiple modelling media. By taking a longer view of how these microbial model systems work, we learn more about how organismal models themselves function and why microbial model systems are particularly valuable material models in the contexts we examine.

\section{Competitive Exclusion Versus Coexistence: Historical and Contemporary Strategies}

Although there is a great deal of modelling work done with large organisms, some of the most prolific and effective modelling with living populations is done with microorganisms. This is especially the case for ecological and evolutionary modelling, where microbial systems offer particularly tractable means for studying dynamical processes at the population level over many generations (a point to which we return in Section 6). In evolutionary biology and ecology, mathematical and material modelling have frequently been combined. In order to gain closer insight into how these modelling strategies work together, we track a trajectory of comparative modelling of the phenomena of competitive exclusion and coexistence. We have chosen this historical lineage of modelling focus and activity because it shows not only how different models have been adjusted in relation to one another, but also how the researchers involved have conceptualized this interaction and adjustment.

\subsection{Gause's Model Comparisons}

Our story begins with Georgii Gause (1910-1986), a mathematically competent experimental biologist who set up microbial model systems of paramecia (bacteriagobbling ciliate protists) to model interspecies competitive dynamics (Gause, 1932; 1934a). These dynamics - the 'struggle for existence' - are central to both evolutionary and ecological theory, and had already been captured abstractly by the Lotka-Volterra mathematical competition model. Gause argued it was imperative to see how well these equations applied to empirical systems: "No mathematical theories can be accepted by biologists without a most careful experimental verification" (Gause, 1934a, p. 59).

Animals, thought Gause, are "too complex" to observe in the wild and impossible to control in the laboratory (Gause, 1934, p. 26). He wanted to "eliminate the complicating influence of numerous secondary factors" in order to "understand the mechanism of the elementary process of the struggle for existence" (Gause, 1934a, p. 92, p.6). To achieve this, he used an experimental system of populations of paramecia species, Paramecium caudatum and $P$. aurelia. ${ }^{4}$ When fed separately on a single species of bacterial prey, each attained a growth equilibrium. But once both paramecia were put together and made to compete for the same bacterial resources, one species became extinct. This demonstration eventually became known as the competitive exclusion principle (Hardin, 1960). The material model system of paramecia demonstrates the same basic mechanisms represented by the Lotka-

\footnotetext{
4 These organisms were already well-established microbial experimental systems (e.g., Woodruff, 1911; 1926). Gause used competing species of yeast as well.
} 
Volterra equations of "indirect" species interactions, which refer to non-predatory competitive relationships or "the struggle for the means of livelihood" (Gause, 1934a, p. 6, p. 3).

However, when Gause introduced into his model system a third level of organism, Didinium, a ciliate predator of the paramecia (thus bringing about a "direct" struggle for existence), he was forced to question the classic Lotka-Volterra formulation of predatorprey relationships and their oscillations (Gause, 1934a, Chapter 6). Why? Because in almost every run of the experimental system, both predator and prey (just a single species of paramecium) went to extinction, "owing to the particular biological adaptations of our predators, which have not been foreseen in the theoretical equations" (Gause, 1934b, p. 17). ${ }^{5}$ The very nature of the equations (deterministic differential equations) was, he thought, inadequate for the relevant system dynamics. Probabilistic equations might be necessary, concluded Gause, in order to capture chance events and the "multiplicity of causes" (Gause, 1934a, p. 124).

Gause then tweaked the experimental conditions of the material model system until a semblance of oscillation occurred. He also made some mathematically remarkable revisions to the Lotka-Volterra model by constructing a limit cycle to predator-prey dynamics with discontinuous differential equations (see Křivan, 2011). ${ }^{6}$ These revisions were not, however, greatly noticed.

\subsection{Explaining coexistence}

In the decades following Gause's pioneering work, many other modellers found coexistence of supposedly competing species, both experimentally (Hutchinson, 1961; Ayala, 1969) and mathematically or computationally (Koch, 1974; McGehee and Armstrong, 1980). ${ }^{7}$ Coexistence violates the very idea of competitive exclusion, which stipulates that different species dependent on identical limiting resources (broadly interpreted as occupying exactly the same niche) cannot coexist indefinitely (unless adaptive change happens, which would mean the niche for the newly adapted organism was different). ${ }^{8}$

One proposal for how periodic equilibria might be achieved is to add predation to standard resource limitation as a second independent limiting factor (Hutchinson, 1961; Levin, 1970). To establish this suggestion more rigorously, population microbiologists Bruce

\footnotetext{
${ }^{5}$ However, Gause did carry out other experimental work with paramecia preying on yeast, and for those model systems did find classic Lotka-Volterra oscillations (Gause, 1935; see Pritchard et al., 2016 for worries about confounding factors that probably produced this result).

${ }^{6}$ Vlastimil Krivan (2011) explains how Gause anticipated more sophisticated predator-prey models of the 1960s (e.g., Rosenzweig and MacArthur, 1963), despite not having the appropriate mathematical tools.

7 Other researchers extensively remodelled the experimental system and the mathematical model (e.g., Luckinbill, 1973; Rosenzweig and MacArthur, 1963).

${ }^{8}$ See Cole (1960) for a scathing critique of the testability and utility of competitive exclusion thus formulated. Hutchinson (1961) sees the same untestability, but does not see this as a problem, merely a property of formal models.
} 
Levin, Frank Stewart and Lin Chao first constructed mathematical models and then their material counterparts (Levin et al., 1977; Chao et al., 1977). They implemented their material model as a chemostat, which is a continuous microbial culturing device that closely regulates the input of nutrients and output of waste (as opposed to non-continuous batch culturing). Chemostats model population growth rates, the dynamics of which are also captured by kinetic growth equations. These equations were initially devised by Jacques Monod (one of the inventors of the physical chemostat) and later elaborated to capture competitive exclusion dynamics in chemostat conditions (see Hsu et al., 1977 for details). However, Monod-based models also failed to predict some experimental system outcomes (Dent et al., 1976), which meant further revisions were needed.

In one of these extensions of the basic model, Levin et al. (1977) used a single nutritional resource for two bacterial populations of Escherichia coli, strain K12 and strain B, plus a virus of $E$. coli, bacteriophage T2. In this system, the bacteriophage plays the role of a predator. The K12 strain of $E$. coli is resistant to the phage, but the B strain is sensitive or vulnerable to its attack. Levin and colleagues first found a stable state for a one-resource, one-consumer (resistant), one-predator situation (unlike Gause). They then extended this finding to the larger system with two consumers, and - in follow-up experimental work - to more complex combinations of populations and trophic levels (Chao et al., 1977). Their amended version of chemostat-based competitive exclusion predicted the equilibrium of all the entities in the model system - both bacteria and the phage. ${ }^{9}$ If there had been no such predator but still two consumers living off a limiting resource, one consumer would have had to become extinct, just as Gause had discovered. The experimental demonstration in the chemostat agreed with the mathematical model, except for one important difference: the material system was stable outside the range predicted by the extended mathematical model (Levin et al., 1977).

The aim of constructing these experimental model systems was to specify the factors that brought about coexistence. In earlier revisions, these equilibria were often "described primarily in terms of the properties of the equation rather than in terms of the biology" (Levin et al., 1977, p. 3). Even though such models were nicely general, they were too nonspecific to predict exactly when coexistence could and should occur, or to explain why it did not. The advantages of the material models were not only that they could specify particular biological conditions, but they were also able to fine-tune the mathematical equations. In addition, experimental data were used to parameterize the equilibria calculations, so the interactions between mathematical and material modelling became even more entangled.

\subsection{Mechanisms of coexistence}

A great deal of other modelling work on these phenomena shuttles similarly back and forth between the equations and the material modelling. Often, it does so to try and explain how coexistence occurs (given that competitive exclusion is the default assumption). One such example is found in an experimental chemostat model of specialist and generalist $E$. coli. In this material model, Daniel Dykhuizen and Maxine Davies (1980) repeatedly found

\footnotetext{
9 The authors described these equilibria as similar to commensalism, in that even though the virus harms the host, it also attacks competitors of the host and thus brings it benefits (Levin et al., 1977).
} 
coexistence of competing strains of $E$. coli. This coexistence had been predicted mathematically by elaborations of Monod's chemostat equations, but the exact biological circumstances in which it would occur were not clear. The experimental system largely agreed with the mathematical predictions but refined them by producing fewer generalists than predicted. To explain this, a new biological mechanism of "resource interference" had to be formulated. It posited that organisms are simply not as effective at multi-resource exploitation as they are at mono-resource utilization (Dykhuizen and Davies, 1980, p. 1213).

Subsequent research efforts worked with systems designed to illuminate the evolutionary dynamics of these ecological phenomena. Organismal model systems were run for longer time periods, and genetic analyses added in order to scrutinize the mechanisms of coexistence. A key series of chemostat experiments in the 1980s found that after several hundred generations, all clonal microbial populations produced polymorphisms (alternative genotypes) and that these polymorphisms were stable: the competing genotype was not able to wipe out the new one (Helling et al., 1987; Rosenzweig et al., 1994).

An even longer analysis was made possible by the model system devised by Richard Lenski's group. This system, a series of bacterial populations maintained as ongoing batch cultures for over 65,000 generations, has modelled numerous evolutionary processes (e.g., Lenski et al., 1991; Lenski, 2011). But as well as this long-running experimental system, Lenski and colleagues have constructed both theoretical and material models in which various manipulations have been carried out specifically to explain coexistence. An early instance of this occurred when Lenski and Susan Hattingh (1986) designed a mathematical model that found an equilibrium between resource-competitive species that were sensitive and insensitive to a particular toxin (i.e., an antibiotic). The modellers showed mathematically the conditions under which such an equilibrium could be realized, and then adduced evidence from chemostat models (some of it in separate work by Hattingh) that supported their conclusions.

In a very different modelling approach 10 years later, Lenski and two other colleagues implemented a material model of coexistence to clarify the mechanisms by which this state was achieved (Turner et al. 1996). They used bacteria from the long-term evolutionary experiment, sampled after the 7000th generation and allowed to diverge for another 1000 generations via recombination. Initially, the researchers had thought the ongoing coexistence of these genotypes would be explained by trade-offs in feeding strategies, with frequency-dependent selection being the mechanism. But this turned out to be an insufficient explanation of the equilibrium, and numerical simulations confirmed the limitations of this explanation. A cross-feeding interaction, whereby each species was better off together than alone during resource limitation, led to the organisms "through their own biological activities, chang[ing] a simple environment into one that is more complex. And this environmental complexity, in turn, allowed for diversity to be stably maintained where it could not otherwise exist" (Turner et al., 1996, p. 2127).

Even when extremely long-term modelling work is carried out (20,000+ generations), coexistence persists (Rozen and Lenski, 2000). This persistence makes clear that coexistence is not an artificial by-product of 'unnatural' ecological and evolutionary conditions in the model. By continuing the model system for even more generations 
(30,000), Lenski and different colleagues (Le Gac et al., 2012) were able to introduce more realistic considerations into evolutionary theorizations of the ecological dynamics of coexistence. Once again, bacterial systems were the key to being able to study these interacting dynamics and timescales. Despite starting with the simplest model possible - a constant environment, one initial genotype, no recombination, and a "focal organism that is much simpler than many others" - complexity in the form of increased diversity still arose (Rozen and Lenski, 2000, p. 33; Turner et al., 1996; Rosenzweig et al., 1994). But just as occurs mathematically, simplifications allow generalizations beyond the original population. Daniel Rozen and Lenski (2000), for example, believed they might be able to generalize their results to the evolution of populations of large sexual organisms as did other researchers of the era (see Adams and Rosenzweig, 2014). In other work, Lenski has also drawn attention to the comparability of numerical simulations (computational models) and microbial model systems, in which each member of the population can be treated as an individual with simple properties in order to understand complex population-level outcomes (e.g., Lenski et al., 2003).

The comparability of these different modelling strategies is highlighted by Ben Kerr and colleagues' (2002) combination of computational models and experimental microbial communities to study the effects of community spatial structure and scale on the maintenance of biodiversity (i.e., coexistence). The focus of both their models is nontransitive communities that lack a competitive hierarchy, such as when three species have a 'rock-paper-scissors' dynamic. This dynamic occurs when each species outcompetes one of the others, but no species outcompetes both of the others. Kerr et al. begin the investigation with a computer simulation of three species representing the rock-paperscissors dynamic. In a lattice-based version of the simulation with minimal dispersal and only local interaction, all three species continued to coexist in clumps. In a second version of the simulation with maximal dispersal (everyone mixing and interacting with everyone else), one species excluded the others. Kerr and colleagues find that localized interactions promote stable coexistence of all three species, but that larger spatial scales of interaction lead to biodiversity loss (one species outcompeting the others).

The paper then describes how in order "to test this conclusion" (Kerr et al., 2002, p. 172), microbial communities were constructed in the laboratory to correspond to exactly the same two scenarios. Solid agar plates with three strains of $E$. coli in a rock-paper-scissors relationship were used to examine the mathematical finding about local interactions. One strain (C) produced a toxin called colicin that harmed a second colicin-sensitive strain (S); $S$, however, had a growth-rate advantage over a third colicin-resistant strain (R); $R$ in turn had a growth-rate advantage over $\mathrm{C}$. The same three strains of $E$. coli in well-mixed flasks of fluid growth media allowed the investigation of dispersal and interaction over (relatively) greater spatial scales. Kerr and colleagues conclude that the results are robust across the computational model and the experimental microbial populations. They interpret this congruence as support for the hypothesis that local interactions - in contrast to dispersal over large spaces - promote stable coexistence.

Kerr and colleagues' sequence of research presents the microbial model system as 'validating' the results from the mathematical model. Their strategy thus echoes the earlier prescription from Gause (1934a, p. 59): about starting with predictions from a mathematical model and then following them up in an experimental model. But as some of 
our other examples demonstrate, researchers could just as easily begin with a material model that was then compared to a mathematical or computational model, where the latter could be seen as 'validating' the results of the former (e.g., Turner et al., 1996; Dykhuizen and Davies, 1980). To understand better how these different modelling strategies work together, we turn to some scientific and philosophical perspectives on model comparison.

\section{Mathematical Models Vis-à-Vis Material Model Systems}

There are two obvious ways in which laboratory microbial populations and computational or mathematical models can work together:

1. Parallel independent modelling: organismal model populations and computational or mathematical population models are used as parallel means to study the same target. This is the dynamic depicted by the right-hand side of Figure 1, where the emphasis is on model-target resemblance assessment.

2. Combined dependent modelling: predictions from mathematical or computational models are evaluated and refined in model organismal populations, and vice versa. This is what the left-hand side of Figure 1 emphasizes.

These two modes of interaction are neither mutually exclusive nor sharply distinguished; cases of modelling practice will sometimes be doing both at once. However, highlighting the difference between the two helps draw out some important points about model-model interactions. The first mode, parallel independent modelling, is most easily understood as the classic strategy of comparing two independent models to the target. Bacterial experimental systems and computational models, for example, are parallel means to gain broad insights into evolutionary processes. This is the standard way to think about multiple kinds of models working together, in which each is studied in order to gain independent insights into the target of evolving populations in the natural world (e.g., Weisberg, 2013). It is usual to describe this procedure as robustness analysis, whereby different models are assessed independently for how well they capture basic phenomena or principles (Levins, 1966; Weisberg, 2006; see Section 5).

Our case study of competitive exclusion and coexistence, however, indicates there are more interactive model-model relationships when model comparison is carried out, and we call this 'combined dependent modelling'. In these situations of strong iterative interplay between models in different media, what exactly is going on? Are the material, computational, and mathematical models of equivalent epistemic status, or are some types of model more fundamental in this interplay? How is the interaction actually assessed? To answer these questions we first look at how modellers themselves discuss these interactions, and then how philosophical accounts of modelling might help make sense of these comparisons.

\subsection{How Modellers Conceptualize Model Interplay}

Amongst the modellers we have been discussing - namely researchers who construct model microorganismal systems in addition to computational and mathematical models to study evolutionary and ecological processes - the general view seems to be that there are some clear differences between mathematical, computational, and material models, and 
that these very differences allow various types of model to work together. Experimental material modelling in multimodel research can motivate modifications to mathematical and computational models; furthermore, it is often strongly expected to do so.

In some discussions, the epistemic equivalence of comparable mathematical and material models is explicitly assumed (e.g., Dykhuizen and Davies, 1980). The chemostat, for example, is sometimes celebrated as "the best laboratory idealization of nature for population studies" because its material processes are "formally equivalent" to natural biological processes (Hsu et al., 1977, p. 366). This claim is made on the basis of the chemostat's inflow-outflow mechanism, which models in a physical medium the same dynamics as the relevant differential equations. ${ }^{10}$ However, there are many other modelling instances, such as Kerr and colleagues' 2002 rock-paper-scissors study, that are framed as 'tests' of the mathematical model by the experimental system. This would seem to indicate that the findings of the experimental models have greater epistemic weight.

Gause, for example, was a strong advocate of the epistemic superiority of material modelling:

There is no doubt that the struggle for existence is a biological problem, and that it ought to be solved by experimentation and not at the desk of a mathematician. But in order to penetrate deeper into the nature of these phenomena, we must combine the experimental method with the mathematical theory (Gause, 1934a, p. 10).

He argued that analysis of the experimental system should come first, methodologically and temporally, and that experiments should be quantitative so that mathematical laws could be built on those quantitative results. However, it is also obvious that his experimental systems were constructed with reference to the equations, with the aim of testing their empirical accuracy.

And as some of our other coexistence examples show, mathematical or computational models are also used for cross-validation and elaboration. For example, numerical simulations in Lenski and Hattingh's (1986) analysis of coexistence showed how the explanation of the coexistence observed in organismal model systems was inadequate. Many modellers suggest that multimedia modelling is needed because the interaction between models turns out to be more complex than can be captured by the language of testing and validation. G. Evelyn Hutchinson (1903-1991) was an influential and widely famed ecologist who worked mostly on multicellular organisms. Some of his research, however, was greatly concerned with explaining why competitive exclusion did not seem to capture the biodiversity of phytoplankton (free-living photosynthesizing marine organisms - usually unicellular). He suggested that:

[mathematical modelling of] competitive exclusion can be used to examine a situation where its main conclusions seem to be empirically false. Just because the theory is

10 Other commentators are more conservative: "A less controversial view of the chemostat $\ldots$ is that it is the best tool available for studying population interactions under controlled conditions" (Fredrickson, 1977, p. 65). 
analytically true and in a certain sense tautological, we can trust in the work of trying to find out what has happened to cause its empirical falsification (Hutchinson, 1961, p. 143).

Levin and colleagues echo this when they argue that by "merely specify[ing] equations [that] do not describe the nature of the interaction[s] ... stability might be merely an artefact of the equations" (Levin et al. 1977, p. 20). This is an issue raised with considerable force by Richard Levins, in his famous 1966 paper motivating robustness analysis. His solution is to "attempt to treat the same problem with several alternative models each with different simplifications but with a common biological assumption" (1966, p. 423). Usually, however, Levins' analysis is thought to apply to model-target relationships rather than the modelmodel relationships we are emphasizing (see Section 5 for further discussion).

Arnie Friederickson, a mathematical modeller of microbial processes, emphasizes the need for a two-way interaction between material and mathematical models but makes it very clear that it is model-model relationships being assessed, and not only model-target resemblances:

Qualitative speculation about [populations] should be checked by the construction and analysis of suitable [mathematical] models, and the models should, in turn, be checked by experiment. [Plus] interesting phenomena predicted by plausible mathematical models of interactions should be searched for experimentally. [And for phenomena for which no experimental work yet exists, m]athematical models of different mechanisms of interaction should be constructed and analysed, and appropriate experimental systems should be set up to test the application of the models (Fredrickson, 1977, p. 84).

Because both material and mathematical models are abstracted and idealized, they indicate to researchers "what is possible" over the course of a research programme (Dykhuizen and Davies, 1980, p. 1213; see also Slobodkin, 1961). This theme of models delineating the possible is also noted by Brendon Bohannan's group of microbial ecologists. They argue that different modelling strategies are not epistemically fixed in the explanatory roles they can play:

The role of theory is to define what is logically possible (given a set of assumptions), the role of the laboratory experiment is to determine what is biologically plausible and the role of the field study is to delineate what is ecologically relevant. Microbial model systems have played all three of these roles: in support of field studies, in support of theory [i.e., equations], and as a bridge between theory and the field (Jessup et al., 2005, pp. 283-284).

Jessup and colleagues go on to suggest that experimental models are particularly useful for 'exploring' mathematical models and determining whether their basic assumptions are right (or plausible). They conclude that microbial model systems are often able to show why a certain mathematical model is not working and how it might be adjusted. For example, spatial structure plays a role in model microbial systems of coexistence (e.g., Kerr et al., 2002), and this recognition has stimulated new mathematical approaches to accommodate spatial dynamics (e.g., Bohannan et al., 
2001; Rainey et al., 2000). We already described how Gause made mathematical innovations on the basis of his experimental system results. These insights into modelmodel comparison and modification also have implications for philosophy of modelling.

\subsection{How Philosophers Conceptualize Model Interplay}

We noted above that philosophy of modelling has not given a great deal of attention to model-model relationships that involve material model systems. However, this may be because there is no such comparison going on in modelling practice. Philosophers might argue that doing an experiment to 'validate' or 'test' a model, as Gause, Kerr and colleagues, and many other researchers have suggested they are doing, means that what we have called 'material model systems' are just real-world phenomena - the target phenomena, in fact (Figure 1). But we have seen repeatedly how simplified, idealized and abstracted these systems are. The laboratory environments usually bear limited resemblance to environments in nature; the microbes themselves are often distant relatives of their non-lab-dwelling ancestors; they are not identical to any populations outside the laboratory; nor do we think they are even straightforward specimens of any such natural populations.

Philosopher of ecology Jay Odenbaugh has likewise emphasized how microcosms (aka 'bottle experiments') are 'stripped down' unnatural systems. But beyond their pragmatic virtues, microcosms have the capacity to "describe the natures, dispositions, or tendencies of objects and how they would behave in simplified circumstances" (Odenbaugh, 2006, p. 727). Rather than expecting nature in a bottle, scientists expect bottled nature to capture key features (but not all features) of their systems of interest. We will suggest in Section 6 that in such 'bottles' (or flasks or plates or chemostats), microbes have some epistemic as well as practical advantages over non-microbes, especially when model comparison is taken into account.

There could also be reasons to think that material model systems are mere abstractions of phenomena in the world. In other words, they might make a necessary step toward modelling, by focusing on certain details of the system of interest and leaving out others (such as relevant environmental conditions), but are not themselves models. Abstract direct representations involve simplified and sometimes generalizable descriptions of phenomena in the natural world (Weisberg, 2007). Finding patterns in datasets would be an illustration of this sort of representation. James Griesemer's (1990) groundbreaking philosophical discussion of material models suggests that some of them are 'direct' but that others function in the normal indirect way of modelling.

Arnon Levy and Adrian Currie (2015) argue along these lines when they raise questions about whether most broad 'model organism' use involves modelling. They define "theoretical modelling" as an activity that "involves a mathematical or mechanistic construct that serves as an analogue of the target", which is then analysed and evaluated for its similarity to the target (Levy and Currie, 2015, p. 331). From their perspective, many discussions of model organisms do not meet these specifications. They argue that model organisms are usually treated as "representational specimen[s] of a broader class, thus allowing extrapolation on the basis of the specimen[s]" (Levy and Currie, 2015, p. 332; see also Katz, 2016 for a similar line of argument). 
However, the majority of the counterexamples Levy and Currie outline are not very similar to the laboratory systems on which we have focused. Levy and Currie's own 'paradigmatic example' of a genuine theoretical model is the Lotka-Volterra equations, which, as we have shown, certainly seem to have organismal model analogues to the mathematical relationships. We believe that Levy and Currie would view Gause's work and the vast amounts of material modelling it has stimulated over many decades as "mechanistic constructs that serve as analogues of the target phenomena" and thus as models (Levy and Currie, 2015, p. 331). This capacity to function as surrogates for much more general target systems is why we do not distinguish in our cases between experimental and model organisms (see Ankeny and Leonelli, 2011).

Another option, especially when there are multiple models of the same phenomenon, is to think that the material version re-describes the original model in a different medium or language. For example, Weisberg discusses a case of the "re-expression" of a mathematical model as an individual-based computational model (Weisberg, 2013, p. 163). Our cases could hypothetically be described in similar terms. Modelling might start with a mathematical model, which is then 're-described' in a new medium (such as computational algorithms or microbial populations). One might think that the 'core' model for competitive exclusion or predator-prey relationships is captured by the equations, and that the algorithm-based model or microbial model system is simply re-implementing that very same model in a computer program or on a laboratory bench (as a 'mere' redescription of the same structures).

We think this is not the best way to understand what is going on in our cases, for two reasons. The first is that any putative re-expression shows features that go well beyond copying. As our examples show, microbial model systems are just as much constructed to represent and simplify natural-world dynamics as any set of equations. Most importantly, material models have lives of their own beyond straightforwardly re-describing the same mathematical structures (Dykhuizen and Davies, p. 1980). By studying a microbial model, a scientist can often reach quite different conclusions from those reached in studying the mathematical model. These materially derived conclusions can directly influence the mathematical representations. In other words, the equations themselves are reinterpreted and restructured in light of the results from the material model. The history of modifications to competitive exclusion and coexistence models show this dynamic very clearly.

A second response could be to claim that model re-expression favours mathematics as the modelling bedrock. Weisberg, for instance, indicates that comparing different sorts of models to a target is ultimately an exercise in comparing them to a mathematical representation of the target, rather than the target itself $(2013$, p. 95). This ordering suggests a certain fundamentality to the mathematics. However, many of our examples and much of the scientific literature do not show mathematical privileging to be true. Numerous ecologists and evolutionists have been more inclined to prioritize what they learn from the material model system findings and alter the mathematics accordingly. As suggested by several of our quotations in Section 4.1, modellers may see the mathematical structures as a flimsy signpost rather than any kind of epistemic bedrock. While the details will of course vary according to the nature of research and its subject matter, there does not seem to be any principled reason always to favour mathematical 
models. Equations are useful descriptions of structural elements; material models including microbes can represent those structures just as effectively or more effectively in certain circumstances.

In all modelling activities, whatever the medium, anything new that is learned concerns the abstracted object of study itself (the particular microbial populations, the computer simulation, etc.). There is always additional work required to draw a broader conclusion that refers back to a target in the natural world (Sprouffske et al., 2012). This is the central activity of all modelling practice and nearly all experimental practice, apart from a rare subset of field experiments. Sometimes this learning occurs linearly, from model to target, but often enough - as our cases show - there are model-model comparisons being made as well. Different models are designed in light of other 'target models' in order to interact with them, and not as mere re-expressions of the original model. Importantly, therefore, the introduction of other model media is not just to represent target phenomena independently but to provide a means of assessing the initial model (dependently). But how is this comparison actually done? To begin to answer this question, we turn to an additional aspect of philosophy of modelling, robustness analysis.

\section{Robustness Analysis and Comparative Modelling}

Philosophical discussions of how models relate to other models have focused on a particular account of robustness analysis. As we noted in Section 4, this procedure is based on multiple models independently homing in on common or core principles to explain target phenomena in the natural world (Levins, 1966; Weisberg, 2006; Weisberg and Reisman, 2008; Lloyd, 2010; see Parker, 2015 for critical questions about how such assessments are implemented). Robustness analysis captures important features of how models relate to their targets, but its operation relies on assumptions of strict model independence that in reality are rarely met (Parker, 2011). Instead of viewing model dependence as a problem, however, our cases of competitive exclusion and coexistence studies highlight how iterations of dependent model comparison can improve and extend model performance.

Robustness analysis has primarily been discussed in relation to mathematical and computational models. Weisberg (2013, pp. 162-163) mentions a failure to implement a concrete model mathematically (the San Francisco Bay model), and notes that mathematical-computational model combinations are the most common. His main example of this more common relationship is the same computational 're-expression' we already mentioned of the Lotka-Volterra predator-prey model (see also Weisberg and Reisman, 2008). Robustness analysis examines supposedly independent models that because of their 'common structures' are able to represent specified properties of the same target system. Even when perturbed, these structures still produce the same or very similar results and are thus deemed robust.

Because robustness analysis involves comparing models in relation to the target system, it indicates that common structures can be realized equally in different media, whether equations, algorithms, or biological populations. It is easy to see how common structures can be found in different mathematical or computational models. One reason that material 
systems are seldom included in discussions of robustness analysis is because their comparability with mathematical or algorithmic structures is not as easily formalized. Our examples show how structural comparisons can also be made between mathematical and material models, even when these are not based on explicitly quantifying and formalizing any structure shared by the two. In particular, many of our cases highlight that the focus of model-model comparison is less about how multiple models agree on their predictions about a target than about how different models fail to agree. This search for disagreement becomes a means of identifying the modifications required in one or the other model. In addition, this 'comparative modelling' occurs without explicit comparison to the relevant targets in the natural world and sidesteps the requirements for independence that come with formal robustness analyses.

Gause's studies of interspecies competition clearly exemplify the comparative modelling process. By studying microbial systems he learned that material representations of the 'direct' struggle for existence did not replicate the findings of the Lotka-Volterra equations, indicating to him that the mathematical model required reformulation. Gause based his mathematical revisions on insights gleaned from microbial systems in the laboratory, and yet had already constructed those microbial systems with reference to the equations. His aim was not only to see where these models agreed, but also to specify how one could be improved in light of its disagreements with the other.

Comparative modelling can thus make an important contribution to assessments of model robustness. As in all robustness analyses, models are compared and their common (and uncommon) parameters, structures, or other features assessed. The key feature of comparative modeling is that it shifts the focus away from model-target comparison to focus instead on model-model relationships, and emphasizes key differences as opposed to commonalities between models. Our cases show us how dependent model comparisons, rather than being an inherent vice of robustness analyses (Parker, 2011), become a virtue that permits iterative model modification. From an analytic point of view, independent models may be highly desirable but difficult to realize, whereas from a practical and historical perspective, the dependent comparison of models is very prevalent and apparently effective.

Another virtue that is apparent from the cases we examine above is that comparative modelling is often qualitative rather than quantitative, whereas discussions of robustness analysis have focused on formalized quantitative comparisons (e.g., Weisberg, 2013). The qualitative comparative modelling we highlight facilitates ready comparisons between material and mathematical models. For example, the same basic dynamical processes of predator-prey interactions are present in both the mathematical models and the microbial systems that feature in Gause's competitive exclusion and Levin and colleagues' chemostat research. The two types of model are compared in virtue of general assumptions about properties, patterns, and processes, and how different media capture these abstract phenomena. Modifications are then carried out to material and mathematical systems, after which fresh inferences can be made about the target system. 


\section{Microbial Model Systems as Special Material Models}

By highlighting these particular features of model-model interplay, we think it is possible to understand better why microbial model systems might be epistemically favoured over non-microbial organismal systems. Microbial models are not the only organisms that function as model systems. Why have we focused on microbial systems rather than, for example, the beetles Odenbaugh (2006) mentions in competitive exclusion and coexistence investigations?

Any general discussion of microbial models first takes into account the tractability of such populations. Small size, rapid generational turnover, limited sexuality, and preservability are the main claims made for microbial tractability (e.g., Lenski et al., 1991; Rainey et al., 2000; Adams and Rosenzweig, 2014). But in addition to the practical tractability associated with small size and short generation time in microbes, our historical narrative also reveals epistemic tractability. Philosophers who have investigated organismal models, both macrobial and microbial, have suggested "representational scope" as the key to how organisms function as models (e.g., Ankeny and Leonelli, 2011, p. 315; Love and Travisano, 2013). We agree with this general point, and want to emphasize how our examples show how this scope is achieved in concert with models in other media. Our cases show many instances in which the structures that make up the microbial model can be compared very effectively to other more abstract structures. For microorganisms, these structures are less obscured, and this simple but crucial point gives a special edge to their representational scope in concert with other models. In larger organisms, many allowances have to be made both to accommodate obfuscating complexity in the model and then rule out its effects.

When Gause said he wanted to "eliminate the complicating influence of numerous secondary factors" in order to get at "the mechanism of the elementary process" (Gause, 1934a, p. 92), he meant this not just for standard practical reasons but also for being able to home in comparatively on structures that could be realized mathematically and materially. The 'eliminations' possible in microbial model systems include removing sex, the complexity of multicellular bodies, environmental variability (including nutritional resources), behavioural complications, and initial genetic diversity (i.e., clonal populations of microbes reproduce from a single genotype, as in Lenski's experimental system). Because such simplifications are possible, the organisms become quantifiable structures in a populational form that can be compared directly to equations.

The mathematical models used in evolution and ecology tend to rely on infinite or huge population sizes to swamp out the effects of stochasticity and drift. Another major advantage of microbial systems is that they allow researchers to study extremely large (though not infinite) laboratory populations that are orders of magnitude greater than can be achieved with larger organisms. Doing so allows for more effective direct comparisons between the microbial models and standard mathematical representations of evolving populations. Similar affinities between microbes and mathematics can be seen in laboratory setups such as chemostats (see Section 3.2). Their precise inflowoutflow systems are physical media that capture the same dynamics as systems of differential equations. These tightly controlled, low-complexity experimental systems of interacting populations are simply unavailable for non-microbial organisms, and their 
availability enables extreme simplification of the material parameters of the model system.

An additional epistemic benefit offered by microbial model systems is that researchers can literally back up and rerun lineages. This is a feature that is otherwise possible only in computer simulations. Because of being able to freeze and revive cultures (bacteria routinely survive storage in minus- 80 and minus-130 degree freezers), microbial populations can function very similarly to an iterated algorithm or set of equations. Larger laboratory organisms, even beetles, cannot be defrosted and revived and 'rerun' in this way. This ability to rerun the evolutionary trajectories of microbial populations an arbitrary number of times in the same way as mathematical or computational models confers a special sort of epistemic tractability on microbial model systems. All of these epistemic benefits explain why the comparison of material and mathematical models has been so frequent amongst microbial population biologists and a little less obvious in other fields.

\section{Conclusions}

Competitive exclusion and coexistence illustrate the epistemic and practical advantages of microbial systems very effectively, but by no means exhaust what can be learned from microbial model system use and its interplay with mathematical modelling. In the historical lineage of examples we describe in Section 3, the microbial model systems are not simply laboratory specimens of the target system, nor are they just another instance of the same model (the relevant mathematical one) in a different medium. Frequently microbial models function to correct the mathematical model's relationship to the target, while also being assessed and refined when compared to the mathematical model. These dependent comparisons across different model media are complementary to accounts of robustness analysis that relate relevant independent models to the ultimate target of inquiry. In many cases, the results of comparative modelling indicate in advance how successful target capture is likely to be.

By examining a historical trajectory of models focused on a particular target phenomenon, competitive exclusion, we show that there are good reasons to be sceptical about distinctions between 'mere' experimental organisms and model organisms, and between experimental systems and material models more generally. Our analysis shows that an epistemic hierarchy of model media does not apply, and that iterative model-based investigation relies on shifting back and forth between models of the same epistemic status. Although we see many particular practical and epistemic advantages to microbial (and especially bacterial) models, none of those advantages apply exclusively to microorganisms. Nevertheless, we suggest that further philosophical work on the history of microbial models will illuminate more acutely the philosophical dimensions of multimodel research.

\section{Acknowledgements}

We thank Yael Ben-Tal, Brett Calcott, Sidney Diamante, Ben Kerr, Carlos Mariscal, Paul Sniegowski, Sam Woolley, the Copenhagen Philosophy of Science group, and Sydney's Theory and Method in Biosciences group for detailed feedback on earlier drafts of the 
paper. MAO's research was supported by the French government via the 'Investments for the future' Programme, IdEx Bordeaux (ANR-10-IDEX-03-02).

\section{References}

Adams, J., \& Rosenzweig, F. (2014). Experimental microbial evolution: history and conceptual underpinnings. Genomics, 104, 393-398.

Armstrong, R. A., \& McGehee, R. (1980). Competitive exclusion. American Naturalist, 115, 151-170.

Beatty, J. 2006. Replaying life's tape. Journal of Philosophy, 103, 336-362.

Bohannan, B.J.M., Kerr, B., Jessup, C. M., Hughes, J. B., \& Sandvik, G. (2002). Trade-offs and coexistence in microbial microcosms. Antonie van Leeuwenhoek, 81, 107-115.

Buckling, A., Maclean, R. C., Brockhurst, M. A., \& Colegrave, N. (2009). The Beagle in a bottle. Nature, 457, 824-829.

Chao, L., Levin B. R., \& Stewart, F. M. (1977). A complex community in a simple habitat: an experimental study with bacteria and phage. Ecology, 58, 369-378.

Cole, L. C. (1960). Competitive exclusion. Science, 132, 348-349.

Dent, V. E., Bazin, M. J., \& Saunders, P. T. (1976). Behavior of Dictyostelium discoideum amoebae and Escherichia coli grown together in chemostat culture. Archives of Microbiology, 109, 187-194.

Desjardins, E. (2011). Historicity and experimental evolution. Biology and Philosophy 26, 339-364.

Downes, S. M. (1992). The importance of models in theorizing: a deflationary semantic view. PSA Proceedings, 1, 142-153.

Dykhuizen, D. E., \& Davies, M. (1989). An experimental model: bacterial specialists and generalists competing in chemostats. Ecology, 61, 1213-1227.

Dykhuizen, D. E., \& Dean A. M. (1994). Predicted fitness changes along an environmental gradient. Evolutionary Ecology, 8, 524-541.

Fredrickson, A. G. (1977). Behavior of mixed cultures of microorganisms. Annual Review of Microbiology, 31, 63-87.

Grigg, R., \& Nguyen, J. (2017). The turn of the valve: representing with material models. European Journal for Philosophy of Science, doi:10.1007/s13194-017-0182-4. 
Garland, T. Jr., \& Rose, M. R. (Eds.). (2009). Experimental evolution: concepts, methods, and applications of selection experiments. Berkeley: University of California Press.

Gause, G. F. (1932). Experimental studies on the struggle for existence. Journal of Experimental Biology, 9, 389-402.

Gause, G. F. (1934a). The struggle for existence. Baltimore: Williams and Wilkins.

Gause, G. F. (1934b). Experimental analysis of Vito Volterra's mathematical theory of the struggle for existence. Science, 79, 16-17.

Gause, G. F. (1935). Experimental demonstration of Volterra's periodic oscillations in the numbers of animals. Journal of Experimental Biology, 12, 44-48.

Giere, R. N. (1988). Explaining science: a cognitive approach. Chicago: Chicago University Press.

Giere, R. N. (2004). How models are used to represent reality. Philosophy of Science, 71: 742-752.

Godfrey-Smith, P. (2006). The strategy of model-based science. Biology and Philosophy, $21,725-40$.

Griesemer, J. R. (1990). Material models in biology. PSA Proceedings, 2, 79-93.

Guala, F. (2002). Models, simulations, and experiments. In L. Magnani, \& N. J. Nersessian (Eds.), Model-based reasoning: science, technology, values (pp. 59-74). NY:

Kluywer/Plenum.

Hardin, G. (1960). The competitive exclusion principle. Science, 131, 1292-1297.

Helling, R. B., Vargas, C. N., \& Adams, J. (1987). Evolution of Escherichia coli during growth in a constant environment. Genetics, 116, 349-358.

Hsu, S. B., Hubbell, S., \& Waltman, P. (1977). A mathematical theory for single-nutrient competition in continuous cultures of micro-organisms. SIAM Journal of Applied Mathematics, 32, 366-383.

Hutchinson, G. E. (1961). The paradox of the plankton. American Naturalist, 95, 137-145.

Inkpen, S. A. (2016). Like Hercules and the hydra: trade-offs and strategies in ecological model-building and experimental design. Studies in History and Philosophy of Biological and Biomedical Sciences, 57, 34-43.

Jessup, C. M., Kassen, R., Forde, S. E., Kerr, B., Buckling, A., Rainey, P. B., \& Bohannan, B. J. M. (2004). Big questions, small worlds: microbial model systems in ecology. Trends in Ecology and Evolution, 19, 189-197. 
Jessup, C. M., Forde, S. E., \& Bohannan, B. J. M. (2005). Microbial experimental systems in ecology. Advances in Ecological Research, 37, 273-307.

Katz, P. S. (2016). 'Model organisms' in the light of evolution. Current Biology, 26, R641R666.

Kerr, B., Riley, M. A., Feldman, M. W., \& Bohannan, B. J. M. (2002). Local dispersal promotes biodiversity in a real-life game of rock-paper-scissors. Nature, 418, 17117-4.

Koch, A. L. (1974). Competitive coexistence of two predators utilizing the same prey under constant environmental conditions. Journal of Theoretical Biology, 44, 387-395.

Knuuttila, T. (2011). Modelling and representing: an artefactual approach to model-based representation. Studies in History and Philosophy of Science, 42, 262-271.

Knuuttila, T., \& Loettgers A. (2016). Modelling as indirect representation? British Journal for the Philosophy of Science, doi:10.1093/bjps/asv055.

Krivan, V. (2011). On the Gause predator-prey model with a refuge: a fresh look at history. Journal of Theoretical Biology, 274, 67-73.

Le Gac, M., Plucain, J., Hindré, T., Lenski, R. E., \& Schneider, D. (2012). Ecological and evolutionary dynamics of coexisting lineages during a long-term experiment with Escherichia coli. Proceedings of the National Academy of Sciences USA, 109, 9487-9492.

Lenski, R. E. (2011). Evolution in action: a 50,000-generation salute to Charles Darwin. Microbe, 6, 30-33.

Lenski, R. E., \& Hattingh, S. E. (1986). Coexistence of two competitors on one resource and one inhibitor: a chemostat model based on bacteria and antibiotics. Journal of Theoretical Biology, 122, 83-93.

Lenski, R. E., Rose, M. R., Simpson, S. C., \& Tadler, S. C. (1991). Long-term experimental evolution in Escherichia Coli. I. Adaptation and divergence during 2,000 generations. American Naturalist, 136, 1315-1341.

Levin, B. R., Stewart, F. M., \& Chao, L. (1977). Resource-limited growth, competition, and predation: a model and experimental studies with bacteria and bacteriophage. American Naturalist, 111, 3-24.

Levin, S. A. (1970). Community equilibria and stability, and an extension of the competitive exclusion principle. American Naturalist, 104, 413-423.

Levins, R. C. (1966). The strategy of model building in population biology. American Scientist, 54, 421-431.

Levy, A. (forthcoming). Modeling without models. Philosophical Studies. 
Levy, A., \& Currie, A. (2015). Model organisms are not (theoretical) models. British Journal for the Philosophy of Science, 66, 327-348.

Lloyd, E. A. (2010). Confirmation and robustness of climate models. Philosophy of Science, $77,971-84$.

Love, A. C., \& Travisano, M. (2013). Microbes modeling ontogeny. Biology and Philosophy, 28, 161-188.

Luckinbill, L. S. (1973). Coexistence in laboratory populations of Paramecium aurelia and its predator Didinium nasutum. Ecology, 54, 1320-1327.

Mäki, U. (2005). Models Are experiments, experiments are models. Journal of Economic Methodology, 12, 303-315.

Matthewson, J. (2011). Trade-offs in model-building: a more target-oriented approach. Studies in History and Philosophy of Science, 42, 324-333.

Morrison, M., \& Morgan, MS. (1999). Models as mediating instruments. In Morrison, M., \& Morgan, MS (Eds.), Models as mediators: perspectives on natural and social sciences (pp. 110-137). Cambridge, UK: Cambridge University Press.

Morgan, M. S. (2005). Experiments versus models: new phenomena, inference and surprise. Journal of Economic Methodology, 12, 317-329.

Odenbaugh, J. (2006). Message in the bottle: the constraints of experimentation on model building. Philosophy of Science, 73, 720-729.

Parke, E. C. (2014). Experiments, simulations, and epistemic privilege. Philosophy of Science, 81, 516-536.

Parker, W. S. (2009). Does matter really matter? Computer simulations, experiments, and materiality. Synthese, 169, 483-496.

Parker, W. S. (2011). When climate models agree: the significance of robust model predictions. Philosophy of Science, 78: 579-600.

Parker, W. S. (2015). Getting (even more) serious about similarity. Biology and Philosophy, 30, 267-276.

Plutynski, A. (2001). Modeling evolution in theory and practice. Philosophy of Science, 68, S225-S236.

Prosser, J. I., Bohannan, B. J. M., Curtis, T. P., Ellis, R. J., Firestone, M. K., et al. (2007). The role of ecological theory in microbial ecology. Nature Reviews Microbiology, 5, 384392. 
Pritchard, J.O., Porter, A.H.M., \& Montagnes, D.J.S. (2016). Did Gause have a yeast infection? Journal of Eukaryotic Microbiology, 63, 552-557.

Rainey, P. B., Buckling, A., Kassen, R., and Travisano, M. (2000). The emergence and maintenance of diversity: insights from experimental bacterial populations. Trends in Ecology and Evolution, 15, 243-247.

Rheinberger, H.-J. (2010). An epistemology of the concrete: twentieth-century histories of life. Durham: Duke University Press.

Rosenzweig, M.L., \& MacArthur, R. H. (1963). Graphical representation and stability conditions of predator-prey interactions. American Naturalist, 97, 209-223.

Rosenzweig, R. F., Sharp, R. R., Treves, D. S., \& Adams, J. (1994). Microbial evolution in a simple unstructured environment: genetic differentiation in Escherichia coli. Genetics $137,903-917$.

Rozen, D.E., \& Lenski, R.E. (2000). Long-term experimental evolution in Escherichia coli. VIII. dynamics of a balanced polymorphism. American Naturalist, 155, 24-35.

Slobodkin, L. B. (1961). Preliminary ideas for a predictive theory of ecology. American Naturalist, 95, 147-153.

Sprouffske, K., Merlo, L. M. F., Gerrish, P. J., Maley, C. C., \& Sniegowski, P. D. (2012). Cancer in light of experimental evolution. Current Biology, 22, R762-R771.

Turner, P. E., Souza, V., \& Lenksi, R. E. (1996). Tests of ecological mechanisms promoting the stable coexistence of two bacterial genotypes. Ecology, 77, 2119-2129

Weber, M. (2014). Experimental modelling in biology: in vivo representation and stand-ins as modeling strategies. Philosophy of Science, 81, 756-769.

Weisberg, M. (2006). Robustness analysis. Philosophy of Science, 73, 730-742.

Weisberg, M. (2007). Who is a modeller? British Journal for the Philosophy of Science, 58, 207-233.

Weisberg, M. (2013). Simulations and similarity: using models to understand the world. Oxford: Oxford University Press.

Weisberg, M., \& Reisman, K. (2008). The robust Volterra principle. Philosophy of Science, $75,106-131$.

Winsberg, E. (2009). A tale of two methods. Synthese, 169, 575-d92.

Woodruff, L. L. (1911). Paramecium aurelia and Paramecium caudatum. Journal of Morphology, 22, 223-3d7. 
Preprint: O’Malley MA, Parke EC (forthcoming) Microbes, mathematics, and models. Studies in History and Philosophy of Science Part A.

Woodruff, L. L. (1926). Eleven thousand generations of Paramecium. Quarterly Review of Biology, 1, 436-438. 